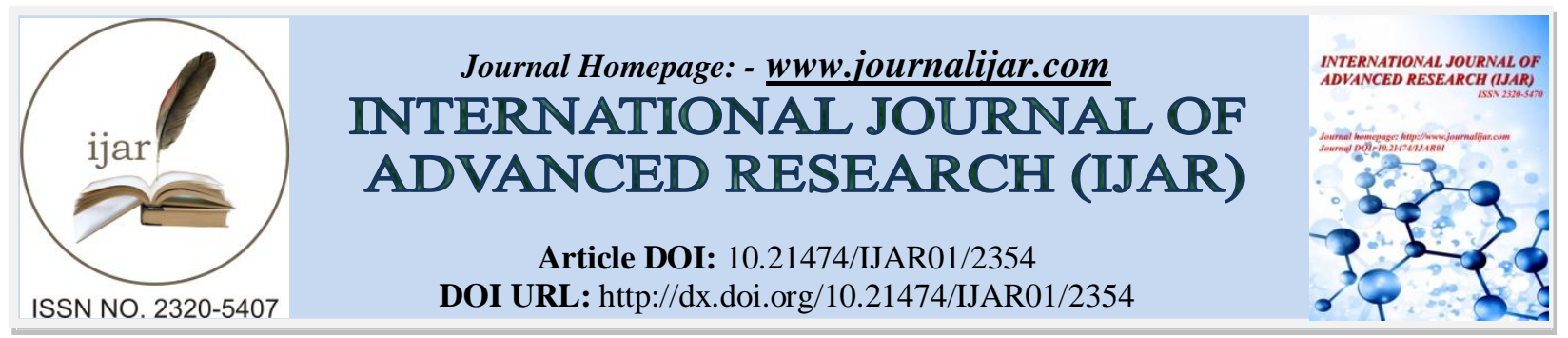

RESEARCH ARTICLE

\title{
L'IMPORTANCE DE LA DIMENSION D'AGE DANS LA FORMATION DE L'INTENTION ENTREPRENEURIALE CHEZ LES CADRES SALARIES : CAS LA REGION SOUSS MASSA DU MAROC.
}

*LAHFIDI Abdelhaq and HOUSSAS M'bark.

Enseignant chercheur ENCG, Universite Ibn Zohr, AGADIR (Maroc), Enseignant chercheur ENCG, Universite Ibn Zohr, AGADIR (Maroc).

\section{Manuscript Info}

Manuscript History

Received: 30 September 2016

Final Accepted: 30 October 2016

Published: November 2016

Key words:-

Intention entrepreneuriale, théorie du comportement planifié, comportement entrepreneurial.

\begin{abstract}
Cette étude se focalise sur l'importance de la dimension d'âge de l'individu et son impact sur sa décision de créer une entreprise et sur la spécificité du comportement des salariés âgés (45 et plus) de la région Souss Massa. En se basant, sur la théorie du comportement planifié, notre papier de recherche présente les résultats d'une étude menée sur un échantillon de 302 cadres salariés dans la région du Souss Massa et montre que l'intention entrepreneuriale varie selon les âges. Cette étude considère que, passé 45 ans, l'âge n'influence plus les trois variables piliers de l'intention entrepreneuriale qui sont l'attitude envers le comportement, le contrôle perçu et les normes subjectives, et plus particulièrement chez les seniors.
\end{abstract}

Copy Right, IJAR, 2016,. All rights reserved.

\section{Introduction:-}

Aujourd'hui, le Maroc est plus entrepreneurial par rapport aux années 1980 et 1990. Cependant il reste insuffisant au regard de l'économie mondiale. Actuellement, la création d'entreprise innovante devient comme un levier pour renforcer l'innovation et la croissance. En effet, les études empiriques montrent que la création et l'accompagnement des entreprises constituent une stratégie importante, notamment pour la croissance et l'emploi (Van Stel et al., 2005) $)^{1}$, la croissance et la productivité (Wennekers et al.,2010) ${ }^{2}$, et aussi pour l'atténuation des inégalités sociales (Fairlie, 2004) $)^{3}$.

Nous nous intéresserons à la création d'entreprise par les salariés de différents âges. En effet, plusieurs constats entrent en vigueur dans ce sens. Le premier constat est l'émergence des jeunes sur le marché du travail, considérés

\footnotetext{
${ }^{1}$.Van Stel, A., Carree, M., Thurik, R., 2005. The Effect of Entrepreneurial Activity on National Economic Growth.

Small Business Economics 24, 311.

${ }^{2}$.Wennekers, S., van Stel, A., Carree, M., Thurik, R., 2010. The Relationship between Entrepreneurship and Economic Development: Is It U-Shaped? Foundations \& Trends in Entrepreneurship 6, 167.

${ }^{3}$ Fairlie, R.W., 2004. Does Business Ownership Provide a Source of Upward Mobility for Blacks and Hispanics?

Entrepreneurship and Public Policy, ed., Doug Holtz-Eakin, Cambridge: MIT Press.
} 
plus entrepreneurial que les seniors (Degeorge et Fayolle, 2011) ${ }^{4}$. Ce constat explique que les jeunes d'aujourd'hui sont de plus en plus motivés par le travail indépendant où ils trouvent plus liberté, de dignité et plus d'occasions pour le développement personnel. Le deuxième constat est relatif aux nouvelles données démographiques au Maroc. En effet, actuellement les retraités représentent la catégorie de la population la plus importante. Les données du haut -Commissariat au plan montrent que 97000 personnes seront admises à la retraite entre 2012 et 2018 avec une moyenne annuelle de 13858 par opposition à une moyenne annuelle seulement de 7400 personnes entre 2004 et 2011. Ces nouvelles données poussent la recherche académique et aussi les experts du domaine à réfléchir sur les possibilités nécessaires à la promotion et l'accompagnement des salariés potentiellement entrepreneurs sans négliger l'accumulation de leur expérience dans la profession ou ils ont passé leurs carrières. En général, cette catégorie de la population se trouve isolée et exclue des cadres d'analyse des professionnels.

Cependant, selon (Safraou et al., 2012) l'engagement entrepreneurial de cette catégorie peut être valorisé comme une réelle opportunité dans une démarche de relance économique et sociale des pays.

Paradoxalement, l'attrait pour la création d'entreprise auprès des jeunes, pose un réel problème de fidélisation des salariés à «haut potentiel » dans un contexte de « guerre des talents».

Notre papier de recherche se structure comme suit : dans un premier temps, nous présentons le cadre théorique de la recherche en se basant sur la théorie du comportement planifié (TCP) et les hypothèses que l'on peut émettre sur l'importance de l'âge sur la décision de créer une entreprise. Ensuite, les résultats de l'étude effectuée sur un échantillon représentatif de la population des cadres salariés de la région de Souss Massa et leur intention de créer une entreprise sont présentés et analysés. Et finalement, nous discuterons les principales caractéristiques de cette étude, en s'intéressant à la spécificité de l'intention entrepreneuriale chez les salariés cadres de la région de Souss Massa ensuite plusieurs recommandations seront formulées et des pistes de recherches seront proposées.

\section{I- lien entre âge et intention entrepreneuriale en se référant à la théorie du comportement planifié:- \\ 1.1 L'intention :-}

De manière générale, L'intention reflète la volonté d'une personne à adopter un certain comportement. Elle suppose de prendre en considération les différents facteurs de motivation qui agissent sur le comportement; c'est un indicateur majeur de la volonté d'un individu à essayer de réaliser un objectif (Kolvereid, 1996) ${ }^{5}$. L'intention reflète aussi bien le désir d'agir que la croyance selon laquelle on va agir. Ce devrait être l'antécédent le plus proche de l'action ou de la tentative de comportement (van Gelderen et al., 2008) ${ }^{6}$.

L'intention et les attitudes sont largement liées aux représentations de soi et de son environnement (Krueger et Carsrud, 1993), ainsi que de ses expériences (Scheiner, 2009; Weick et Karl, 2015). L'intention est une perception qui permet d'imaginer la création d'entreprise comme une voie de carrière possible. L'intention ne constitue pas une action mais une voie vers la réalisation. Elle représente une pensée à développer. C'est une croyance sur le futur envisagé et préféré, mais qui n'est pas encore certain.

\subsection{La théorie du comportement planifié :-}

La théorie du comportement planifié envisagée par Ajzen $(1988,1991)^{7}$ permet de prédire et d'expliquer le comportement d'un individu en différentes situations en analysant l'intention qu'a ce dernier de faire quelque chose. Elle s'appuie particulièrement sur la théorie de l'action raisonnée (Ajzen et Fishbein, 1969).

Plusieurs auteurs (Autio et al., 2014; Davidsson, 1995; Kolvereid, 1996; Krueger et al., 2000; Tkachev et Kolvereid, 1999) suggèrent l'utilisation de la théorie du comportement planifiée de Ajzen pour prédire et expliquer le comportement entrepreneurial. La TCP permet de prédire l'intention entrepreneuriale, puis l'action. La variable principale de ce modèle est l'intention. La création d'une entreprise est un comportement planifié et, donc

${ }^{4}$ Degeorge, J.-M., Fayolle, A., 2011. Les étudiants français ont-ils la fibre entrepreneuriale? Entreprendre \& Innover 9-10, 21-28. doi:10.3917/entin.009.0021

${ }^{5}$ Kolvereid, L., 1996. Prediction of employment status choice intentions. Entrepreneurship: Theory \& Practice 21, 47.

${ }^{6}$ van Gelderen, M., Brand, M., van Praag, M., Bodewes, W., Poutsma, E., van Gils, A., 2008. Explaining entrepreneurial intentions by means of the theory of planned behaviour. Career Development International 13, $538-559$

${ }^{7}$. Ajzen, I., 1991. The theory of planned behavior. Organizational Behavior and Human Decision Processes 50, 179211. doi:10.1016/0749-5978(91)90020-T 
intentionnel. Dans ces hypothèses, l'intention apparaît comme l'élément fondamental de prédiction du comportement que les attitudes, les croyances ou d'autres variables psychologiques (Krueger et Carsrud, 1993) ${ }^{8}$. Plus l'intention de s'impliquer est forte, plus l'individu a des chances de réussir l'objectif qu'il s'est donné, notamment la création d'une entreprise.

La théorie du comportement planifié considère que l'intention est fonction de trois antécédents :-

- L'attitude envers le comportement (attitude toward the behavior) : Cette attitude dépend des croyances relatives aux conséquences du comportement et de la valeur accordée à ces conséquences. Il s'agit de l'évaluation favorable ou défavorable que l'individu a envers le comportement concerné.

- Les normes subjectives (subjective norm) : Cet antécédent s'inspire de la pression sociale perçue pour réaliser (ou pas) un comportement (Ajzen, 1991). Il est engendré par les croyances et les comportements du contexte social de l'individu (conjoint de la personne, famille, amis, etc.).

- Le contrôle comportemental perçu (perceived behavioral control) : Il renvoie à la perception des opportunités ainsi que les ressources disponibles pour la réalisation du comportement. Il s'agit de la difficulté ou, au contraire, de la capacité pour l'individu d'adopter ou non ce comportement.

La figure suivante retrace l'articulation entre ces différentes notions.

Figure 1 : La théorie du comportement planifié

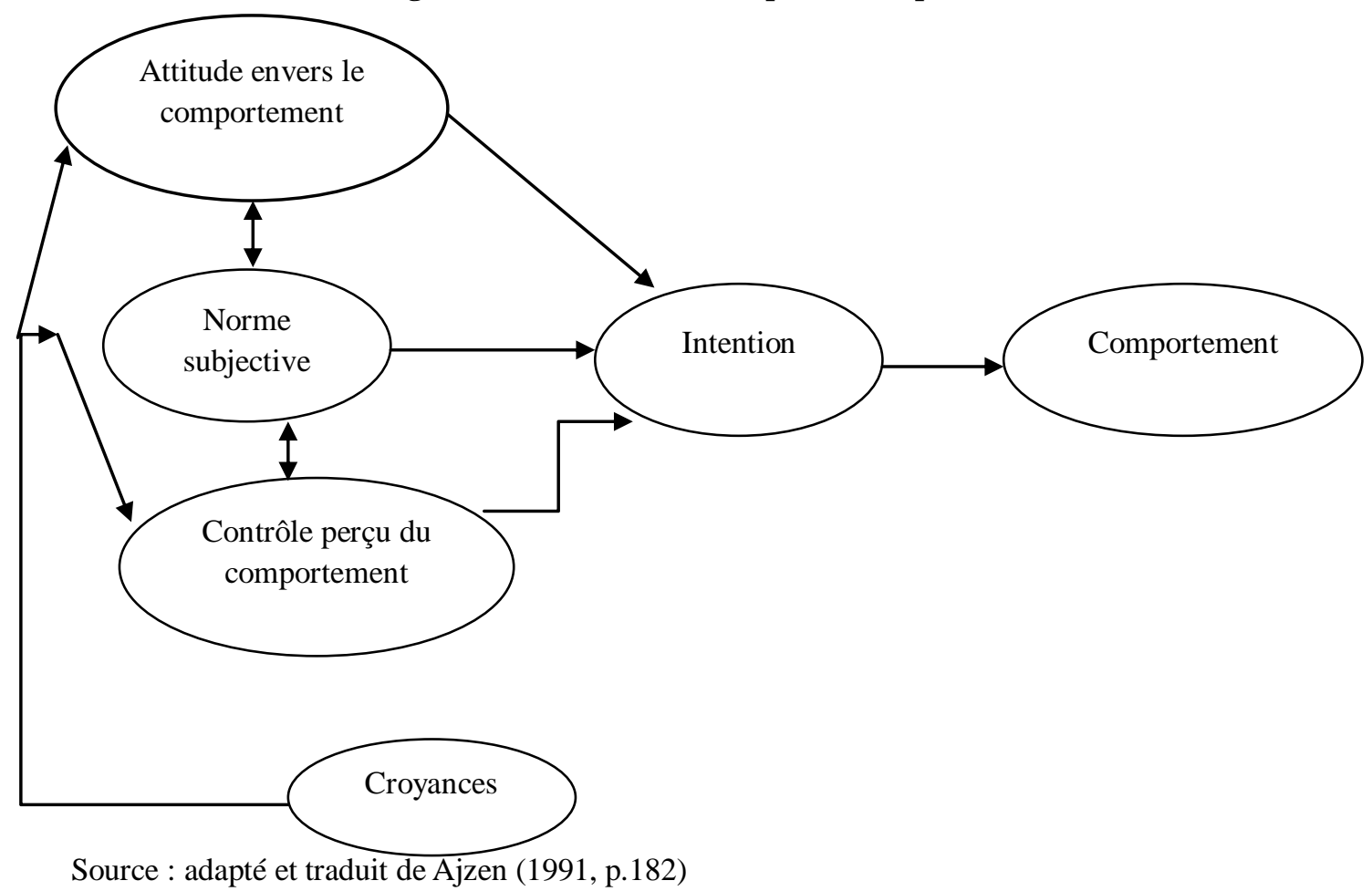

\subsection{L'âge et l'intention entrepreneuriale :-}

Pour plusieurs recherches la TCP est considérée comme une référence théorique. Cependant, certaines études empiriques ont donné des résultats divergents (Ogden, 2003) ${ }^{9}$. En effet, certaines recherches ont montré l'inexistence d'un rôle des normes subjectives, d'autres ont établi la non importance du rôle prédictif du contrôle

${ }^{8}$ Krueger, N.F., Carsrud, A.L., 1993. Entrepreneurial intentions: Applying the theory of planned behaviour. Entrepreneurship \& Regional Development 5, 315

${ }^{9}$ Ogden, J. [b1] (analytic), 2003. Some problems with social cognition models: A pragmatic and conceptual analysis (English). Health psychology (Hillsdale, N.J.) 22, 424-428. 
comportemental perçu, et d'autres ont constaté un rôle nul pour les attitudes. Ces différences des résultats s'expliquent par le le fait que le facteur relatif des attitudes, des normes subjectives et des perceptions du contrôle comportemental pour la prédiction des intentions varient d'un comportement à un autre (Ajzen et Fishbein , 2004) ${ }^{10}$ . Pour ces auteurs, les trois antécédents n'expliquent pas exhaustivement l'intention entrepreneuriale. Parfois un ou deux antécédents sont suffisants pour avoir cette intention. Cependant, la TCP ne précise pas le degré d'impact de chaque facteur sur l'intention entrepreneuriale, sur quelle population et dans quel contexte.

De plus, la théorie du comportement planifié, limite les déterminants de l'intention aux attitudes, aux normes subjectives et au contrôle comportemental perçu alors qu'il peut y avoir d'autres facteurs influençant les comportements comme par exemple les facteurs de personnalité, culturels et démographiques. Ne pas intégrer d'autres antécédents ou les antécédents clés pourrait être la cause d'avoir des résultats différents obtenus par les recherches antérieures ayant utilisé la TCP. Cette théorie est fortement utilisée dans les recherches sur l'intention entrepreneuriale en particulier chez les étudiants, dans les universités américaines (Krueger, 1993 ;

Krueger et al., 2000), dans les écoles de commerces les mieux classées en France (Fayolle et al.,2006 2006, Fayolle et Gally, 2009), en Russie (Tkachev et Kolvereid, 1999) ou encore dans les programmes bachelors en Norvège (Kolvereid, 1996), prouvant ainsi que le modèle porté par cette théorie est applicable dans différents contextes, dont celui des étudiants. Cependant, nous penserons que les résultats provenant de ces recherches ne peuvent pas être généralisables sur une population de salariés, ces derniers peuvent présenter une spécificité différente. Ceci s'explique par le faite que l'intention entrepreneuriale est étroitement liée au processus de socialisation de l'individu et aux réseaux tant professionnels que personnels dans lesquels il évolue (Minniti et Lévesque, 2010) ${ }^{11}$. Ces auteurs ont souligné que l'intention entrepreneuriale est d'autant plus forte que l'individu est âgé. Le vieillissement joue un rôle considérable au niveau des changements dans les comportements des individus. Selon (Tornikoski et al., 2012) $)^{12}$, avec l'avancement de l'âge, les individus tendent à adopter un comportement plus conservateur. Dans ce cadre, certains chercheurs ont montré que l'âge a un effet négatif sur les intentions entrepreneuriales. Cette constatation de relation linéaire entre âge et intention est contestée par d'autres observations ou l'âge joue un rôle inversement proportionnel dans la formation de l'intention entrepreneuriale (Alba-Ramirez, 1994 ; Bates, 1995 cité par Tornikoski et al., 2012).

En vue de ces résultats contradictoires, nous pensons à étudier la façon dont l'âge doit être modélisé sur une population de salariés. Nous pouvons avancer que l'âge peut jouer un rôle considérable dans le construit de l'intention entrepreneuriale.

Nous pouvons émettre comme hypothèse dans cette recherche, que l'âge modère la relation entre les trois composantes de la TCP (attitude envers le comportement, normes subjectives perçues et contrôle comportementale perçu) et l'intention entrepreneuriale. Pour la première composante de la TCP (attitude envers le comportement), nous avançons que l'influence de l'attitude envers le comportement serait plus forte chez les jeunes salariés que chez les seniors. En effet, selon (Fayolle et al., 2012), les individus de la génération Y seraient plus entrepreneuriale que les générations précédentes. Plusieurs travaux de recherche mettent en évidence la particularité de cette génération notamment ses caractéristiques et ses attentes envers la création de l'entreprise. Plusieurs attitudes caractérisent cette génération comme par exemple : l'indépendance, l'estime en soi, le besoin d'accomplissement et le pouvoir (Brillet et al., 2013 ; Bourhis et Chenevert, 2010), les jeunes cadres salariés recherchent des perspectives d'épanouissement et n'hésitent pas de quitter l'entreprise au moment ou ils trouvent une opportunité à l'extérieur (Dejoux et Wachtler, 2011).

Les jeunes ont certainement une vision plus rationnelle de l'entreprise, et non plus affective c'était le cas pour les générations précédentes. Tous ces facteurs expliquent en grande partie que l'entrepreneuriat attire de plus en plus les jeunes.

\footnotetext{
${ }^{10}$ Ajzen, I., Fishbein, M., 2004. Questions Raised by a Reasoned Action Approach: Comment on Ogden (2003). Health Psychology 23, 431-434.

${ }^{11}$ Minniti, M., Lévesque, M., 2010. Entrepreneurial types and economic growth. Journal of Business Venturing 25, 305-314.

${ }^{12}$ Tornikoski, E.T., Kautonen, T., Le Loarne, S., 2012. Le rôle de l'âge dans l'intention entrepreneuriale. Revue française de gestion 227, 95-109
} 
La population des seniors semble différente de celle des jeunes. Il serait plus loyale envers l'entreprise et la hiérarchie. Nous pouvons avancer que les seniors sont moins attirés par l'entrepreneuriat car ils appartiennent à une génération qui n'a pas bénéficié de la sensibilisation à l'entrepreneuriat pendant leurs études aux universités (Fayolle et Gailly, 2015) ${ }^{13}$.

Pour les seniors, cette population semble différente par rapport à la population des jeunes. Ils seraient plus loyaux envers l'entreprise et la hiérarchie à l'entrepreneuriat à l'école (Fayolle et Gailly, 2015).

Sur la base de cette revue de littérature, nous formulons l'hypothèse 1 de la manière suivante :

H1. L'âge modère la relation entre l'attitude envers le comportement et l'intention entrepreneuriale chez les salariés ; la relation entre l'attitude et l'intention entrepreneuriale est plus forte chez les jeunes salariés que chez les seniors salariés.

Paradoxalement, nous pensons que l'impact du facteur du contrôle perçu du comportement sur l'intention entrepreneuriale serait plus grand chez les salariés âgés que les jeunes. Plusieurs arguments expliquent cette constatation. Ils ont atteint l'âge où les contraintes financières sont à leur minimum ou n'existent pas, les crédits sont achevés, pas de charges d'enfants (Safraou et al., 2012). Le cumul de savoir faire et d'expérience permettent aux seniors d'apercevoir la création d'entreprise de manière plus faisable que les jeunes salariés. Sans oublier les contacts et les relations qui ont eu avec les professionnels qui agissent positivement sur la décision de création d'entreprise.

Ces arguments nous aident à formuler 1 deuxième hypothèse comme suit :

H2. L'âge modère la relation entre le contrôle perçu du comportement et l'intention entrepreneuriale : l'intensité de la relation entre la faisabilité perçu du comportement et l'intention entrepreneuriale est plus forte chez les personnes âgées que chez les jeunes.

Et finalement, nous discutons dans cette recherche que l'effet des normes subjectives sur l'intention entrepreneuriale baisserait avec l'âge. Les jeunes salariés sont souvent attachés à leur famille, ils ont souvent tendance à consulter les parents pour les projets qu'ils entreprennent. Les parents ne seraient pas tellement favorables pour l'entrepreneuriat, car elle présente un facteur de risque important pouvant ruiner la carrière de leurs enfants. A cet effet, nous émettons la troisième hypothèse comme suit :

H3. L'âge est un facteur modérateur entre les normes subjectives perçues et l'intention entrepreneuriale : l'intention entrepreneuriale est plus guidée par les normes subjectives chez les jeunes salariés que les salariés plus âgés.

Nous résumons nos hypothèses dans cette étude dans la figure suivant :

Figure 2 : l'âge et les 3 conditions de l'intention entrepreneuriale

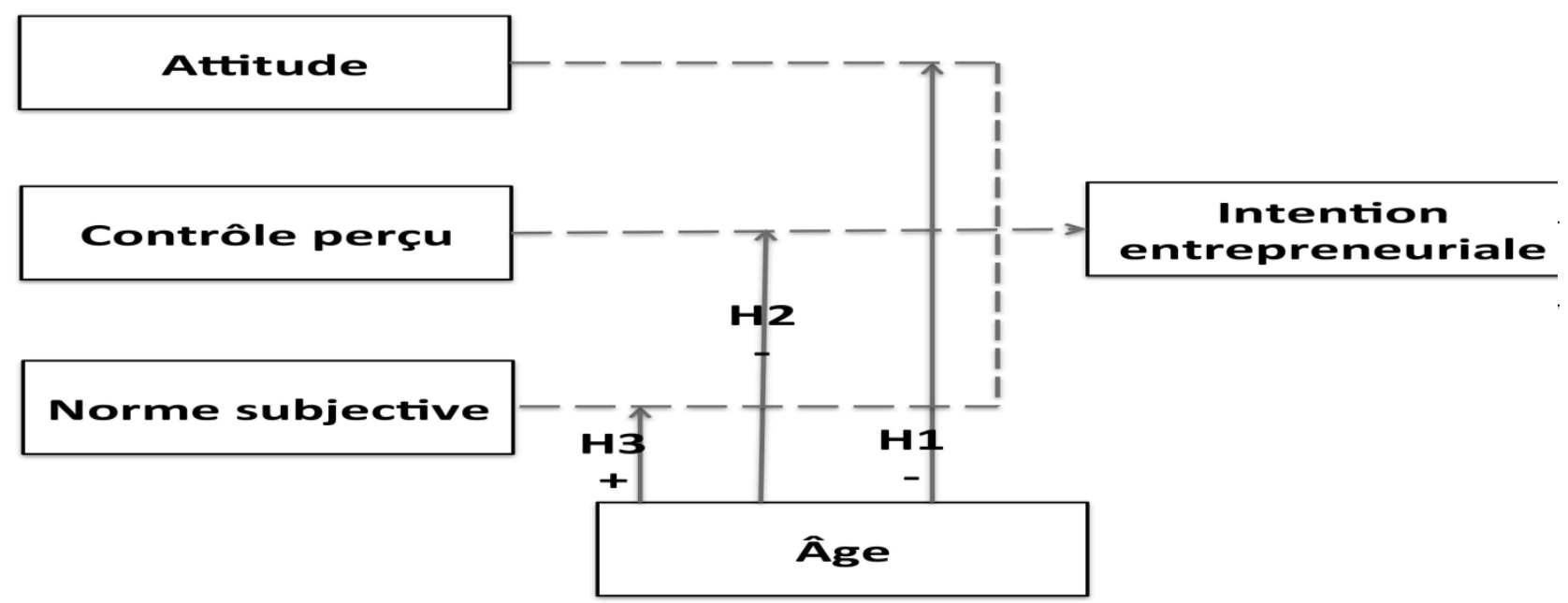




\section{Méthodologie:-}

\subsection{Collecte de données :-}

Pour des raisons pratiques d'accessibilité à l'information et de coût, nous avons choisi un échantillon de convenance composé de 300 salariés cadres exerçant dans des secteurs différents. Le recours aux individus opérant dans des secteurs distincts permet d'éviter un écart relatif à l'échantillon de convenance. Nous nous intéresserons aux salariés cadres occupant un poste de responsabilité dans l'entreprise ou l'établissement où ils travaillent. Il s'agit d'un échantillon par choix raisonné qui permet de retenir les individus qui appartiennent à la population des salariés cadres de la région de Souss Massa.

Au départ, le questionnaire a été envoyé par voie électronique à 750 salariés cadres relevant des différents secteurs comme : enseignement supérieur, pharmacie, immobilier, banque, maritime, industries, agricole, télécommunications, services et travaux publics. Ensuite nous avons reçu 302 réponses qui vont constituer la base de notre étude. Au travers des analyses en composante principale (ACP) et de test de fiabilité interne, nous avons par la suite pu tester et purifier les échelles de mesure.

Les réponses ont été reportées sur des échelles de Likert (1932) ${ }^{14}$ à 7 niveaux (allant de «pas du tout d'accord »à « tout à fait d'accord »).

En ce qui concerne l'intention entrepreneuriale, six mesures ont été retenues : jamais chercher des opportunités de démarrage d'entreprise, économiser de l'argent pour démarrer une nouvelle entreprise, lecture de documents pertinents sur la façon de mettre en place une nouvelle entreprise,

Intention entrepreneuriale, mettre de plans pour démarrer l'entreprise, le temps à passer pour étudier le démarrage d'une nouvelle entreprise, avoir l'intention de démarrer une nouvelle entreprise dans le futur.

Pour l'attitude envers le comportement, ou l'attrait pour la création d'entreprise, une seule question a été posée «l'idée de créer votre entreprise vous semble...» pour mesurer l'attrait sur une échelle allant de pas du tout attractive à tout à fait attractive. Le résultat des tests de fiabilité permet de valider l'échelle (Variance expliquée $=74 \%, \alpha$ de Cronbach $=0,84)$.

En ce qui concerne les normes subjectives, nous avons utilisé l'échelle de (Kolvereid Lsaksen, 2006) ${ }^{15}$ pour mesurer les opinions des individus à propos du choix pour la carrière d'entrepreneur. Dans le questionnaire, nous avons retenu quatre groupes d'individus appartenant à l'environnement social de l'individu qui sont : famille, amis, professeurs et autres groupe important. L'individu de l'échantillon doit préciser l'importance et l'impact de chaque groupe sur sa perception de créer une entreprise. Les échelles proposées allant de « extrêmement défavorable » à " extrêmement favorable » et de «Pas du tout important » à «Extrêmement important ». Le résultat des tests de fiabilité permet de valider l'échelle (Variance expliquée $=72 \%, \alpha$ de Cronbach $=0,82$ ).

Et en fin, pour le contrôle perçu du comportement qui renvoie au degré de capacité de l'individu de penser à pouvoir mener à bien la création d'une entreprise, nous avons utilisé l'échelle de (Emin, 2004) composée des quatre questions suivantes : Vous vous sentez capable de mener à bien un projet de création d'entreprise, vous pensez maîtriser personnellement le processus de création d'une entreprise, vous semble possible de monter un projet de création et d'assurer son aboutissement et vous avez confiance en votre capacité à réussir une création d'entreprise. Le résultat des tests de fiabilité permet de valider l'échelle (Variance expliquée $=81 \%$. $\alpha$ de Cronbach $=0,89$ ).

\subsection{Les variables de contrôle :-}

Une revue de littérature sur le sujet permet de retenir quatre variables de contrôle: âge, genre, secteur d'activité et rémunération. Selon (Mars et al., 2014 ; Wilson et al., 2007) l'intention entrepreneuriale a toujours été associée avec le genre. En effet il a apparu que les hommes sont plus aventureux que les femmes dans leur carrière. Dans le même sens la variable âge a été prise en raison de son impact sur la décision de l'individu de créer une entreprise.

\footnotetext{
${ }^{14}$ Likert, R., 1932. A technique for the measurement of attitudes. Archives of Psychology 22, 1-55.

Maâlaoui, A., Fayolle, A., Castellano, S., Rossi, M., Safraou, I., 2012. L'entrepreneuriat des seniors. Revue française de gestion 227, 69-80. doi:10.3166/RFG.227.69-80

${ }^{15}$ Kolvereid, L., 1996. Prediction of employment status choice intentions. Entrepreneurship: Theory \& Practice 21, 47.
} 
Cependant la relation entre l'âge et la probabilité de créer une entreprise serait largement controversée. L'avancement de l'âge assure que l'individu est devenu plus expérimenté, chose qui augmente la chance de créer son propre entreprise. Paradoxalement, lorsque les individus vieillissent, leur coût d'opportunité devient très élevé avec l'augmentation du revenu, chose qui diminue la probabilité de penser à créer leurs propres entreprises. Nous proposons que la variable revenu est négativement corrélée avec l'intention entrepreneuriale (Long, 1978). La variable secteur d'activité a impact non négligeable sur l'intention entrepreneuriale. En effet, certains secteurs (assurance, banque, informatique...,) donnent plus de chance aux individus de penser à la création d'entreprise comparativement aux autres secteurs (enseignement supérieur, industrie, administration publique...,).

Pour identifier les structures dans les données, absence de colinéarité ou pas, nous avons calculé les coefficients de corrélation de Pearson entre les variables indépendantes (explicatives) mentionnés dans le tableau ci-dessous. La lecture de ce tableau permet de remarquer que tous les coefficients de corrélation sont inférieurs à 0,8 qui est une limite à partir de laquelle il commence à apparaître un sérieux problème de multi-colinéarité. Nous avons calculé le facteur d'inflation de la variance ou le VIF (variance inflation factor) qui est l'inverse de la tolérance, qui permet de diagnostiquer la présence ou non d'une colinéarité entre les variables explicatives. Le VIF le plus grand ici est de 1,78 qui est inférieur à 10 comme étant la limite à partir de laquelle il commence à y avoir un sérieux problème de multi-colinéarité (Arcimoles et Trébucq, 2005 ; Myers, 1990). Ces tests de corrélation et de VIF montrent l'absence de problème de multi-colinéarité entre les variables explicatives.

Tableau 1 : Moyennes, écarts-type et corrélations $(\mathrm{N}=302)$

\begin{tabular}{|c|l|l|l|l|l|l|l|l|}
\hline Variables de contrôle & 1 & 2 & 3 & 4 & 5 & 6 & 7 & 8 \\
\hline$\bullet \quad$ Sexe 1 & 1 & $-0,183$ & 0,19 & $-0,087$ & $-0,23^{* *}$ & $-0,013$ & $-0,009$ & $-0,17^{* *}$ \\
\hline$\bullet \quad$ Âge 2 & $-0,183^{* *}$ & 1 & 0,125 & 0,289 & $-0,11^{*}$ & $-0,023$ & 0,023 & 0,172 \\
\hline$\bullet \quad$ Secteur d'activité 3 & 0,19 & 0,125 & 1 & $-0,082$ & $-0,16^{* *}$ & $-0,090$ & $-0,121$ & $-0,13^{*}$ \\
\hline$\bullet \quad$ Revenu 4 & $-0,087$ & 0,289 & $-0,082$ & 1 & $-0,078$ & $-0,004$ & 0,088 & 0,076 \\
\hline Variable dépendante & & & & & & & & \\
\hline $\begin{array}{l}\text { Intention } \\
\text { entrepreneuriale 5 }\end{array}$ & $-0,23^{* *}$ & $-0,11^{*}$ & $-0,16^{* *}$ & $-0,078$ & 1 & 0,592 & 0,025 & 0,604 \\
\hline Variables indépendantes & & & & & & & & \\
\hline$\quad$ Attitude 6 & $-0,013$ & $-0,023$ & $-0,090$ & $-0,004$ & 0,592 & 1 & 0,316 & 0,542 \\
\hline$\quad$ Normes subjectives 7 & $-0,009$ & 0,023 & $-0,121$ & 0,088 & 0,025 & 0,316 & 1 & 0,272 \\
\hline$\quad$ Contrôle perçu 8 & $-0,17^{* *}$ & 0,172 & $-0,13^{*}$ & 0,076 & 0,604 & 0,542 & 0,272 & 1 \\
\hline Moyenne & 1,32 & 1,32 & 1,32 & 1,32 & 1,32 & 1,32 & 1,32 & 1,32 \\
\hline Ecart-type & 0,485 & 0,485 & 0,485 & 0,485 & 0,485 & 0,485 & 0,485 & 0,485 \\
\hline
\end{tabular}

$* \mathrm{p}<0,05, * * \mathrm{p}<0,01, * * * \mathrm{p}<0,001$.

\section{Résultats:-}

Plusieurs modèles statistiques ont été construits pour tester les hypothèses retenues dans notre étude. Le tableau 2 montre les résultats de régression obtenus.

Le modèle 1 (voir tableau 2) est un modèle de corrélation linéaire qui montre les effets des variables de contrôle sur l'intention entrepreneuriale.

Nous remarquons que le genre a un impact négatif qui n'est pas tellement significatif sur l'intention entrepreneuriale. Ce qui montre que les hommes sont plus attirés vers la création d'entreprise que les femmes $(\mathrm{r}=$ $0,12, \mathrm{t}=-4,9, \mathrm{p}<0.001)$.

Nous constatons aussi que l'âge a un effet négatif sur l'intention entrepreneuriale, ceci signifie que l'intention entrepreneuriale diminue avec l'âge $(\mathrm{r}=-0,11, \mathrm{t}=-1,9, \mathrm{p}<0.05)$.

Les résultats de la régression montrent que le revenu a un impact négatif sur l'intention entrepreneuriale $(\mathrm{r}=-0,078$, $\mathrm{t}=-2,1, \mathrm{p}<0.05$ ). Nous pouvons dire que l'intention de créer une entreprise diminue avec l'augmentation du revenu.

Dans le modèle 2, nous cherchons à savoir l'influence des variables explicatives (Attitude, normes subjective, contrôle perçu) sur l'intention entrepreneuriale.. Une simple lecture nous montre que l'attitude agit positivement l'intention entrepreneuriale $(\mathrm{r}=0,592, \mathrm{t}=7,2, \mathrm{p}<0.001)$. De même, le contrôle perçu a un impact positif sur 1 'intention entrepreneuriale $(r=0,604, t=6,72, p<0.001)$. 
Et finalement, nous remarquons que ce modèle ne permet de déduire de relation significatif entre les normes subjectives et l'intention entrepreneuriale, chose qui nous permet de dire que ces deux variables sont peut être indépendantes $(\mathrm{r}=0,025, \mathrm{t}=0,844)$.

Tableau 2 : la régression hiérarchique par la méthode OLS $(\mathrm{N}=302)$

\begin{tabular}{|l|l|l|l|}
\hline \multirow{2}{*}{ Constante } & \multicolumn{3}{|c|}{ Variable dépendante : intention entrepreneuriale } \\
\cline { 2 - 4 } & \multicolumn{1}{|c|}{ Modèle 1 } & \multicolumn{1}{|c|}{ Modèle 2 } & Modèle 3 \\
\hline Variable de contrôle & - & $5,523^{* * *}$ & $3,863^{* * * *}$ \\
Sexe & $-0,23 * *(-4,816)$ & $-0,146^{* * * *}(-3,93)$ & $-0,142^{* * *}(-\mathbf{3 , 8 1})$ \\
Age & $-0,11^{*}(-1,518)$ & $-0,138^{* * *}(-3,86)$ & $-0,242(\mathrm{n} . \mathrm{s})(-1,73)$ \\
Secteur d'activité & $-0,16(\mathrm{n} . \mathrm{s})(-1,74)$ & $-0,028(\mathrm{n} . \mathrm{s})(-0,75)$ & $-0,029(\mathrm{n} . \mathrm{s})(-0,96)$ \\
Rémunération & $-0,078(\mathrm{n} . \mathrm{s})(-1,61)$ & $-0,098^{*}(-2,53)$ & $-0,087 *(-2,726)$ \\
& & & \\
\hline Les effets principaux & & $0,352 * *(8,262)$ & $0,340 *(1,97)$ \\
Attrait & & $0,297 * *(6,675)$ & $0,381 *(2,042)$ \\
Capacité perçu & & $0,03(\mathrm{n} . \mathrm{s})(0,84)$ & $0,165(\mathrm{n} . \mathrm{s})(-1,262)$ \\
Normes subjectives & & & \\
\hline
\end{tabular}

$*_{p}<0,05, * * p<0,01, * * * p<0,001$. N.s : Non significatif, $t$-statistic entre parenthèses

Les résultats de la régression dans le modèle 2 postulent que l'attitude a un impact positif sur l'intention entrepreneuriale $(\mathrm{r}=0,352, \mathrm{t}=8,662, \mathrm{p}<0.001)$. De même, le contrôle perçu agit positivement sur l'intention entrepreneuriale $(\mathrm{r}=0,297 \mathrm{t}=6,875, \mathrm{p}<0.001)$. Par contre, nous $\mathrm{n}$ 'avons pas pu établir une relation significative entre les normes subjectives et l'intention entrepreneuriale $(r=0,03, t=0,844)$. Nous constatons que, dans le modèle 3 , les effets interactifs des trois composantes (attitude, norme subjective, contrôle perçue) avec l'âge ne sont pas tellement significatifs. Cependant, ceci ne signifie pas qu'il n'y a pas d'effet de modération de la variable âge. Suivant les recommandations de Brambor et al. (2006) -cité par Tornikoski et al. (2012), les résultats des régressions fournissent une information limitée pour interpréter de manière claire les interactions. Il explique que lorsque la variable d'interaction est continue, il devient alors nécessaire de calculer les effets marginaux de chacun des trois antécédents de l'intention entrepreneuriale pour chaque âge. A travers de cette régression on peut conclure que l'effet de la norme subjective est relativement indépendant de l'âge, par contre les effets des deux autres variables attitudes et contrôle perçu - varient clairement en fonction de l'âge (voir les figures 4 et 5). Les hypothèses 1 et 2 sont validées mais l'hypothèse 3 est rejetée.

Figure 3 : Effet marginal de l'âge sur l'intention entrepreneuriale (Intervalle de confiance : $95 \%$ )

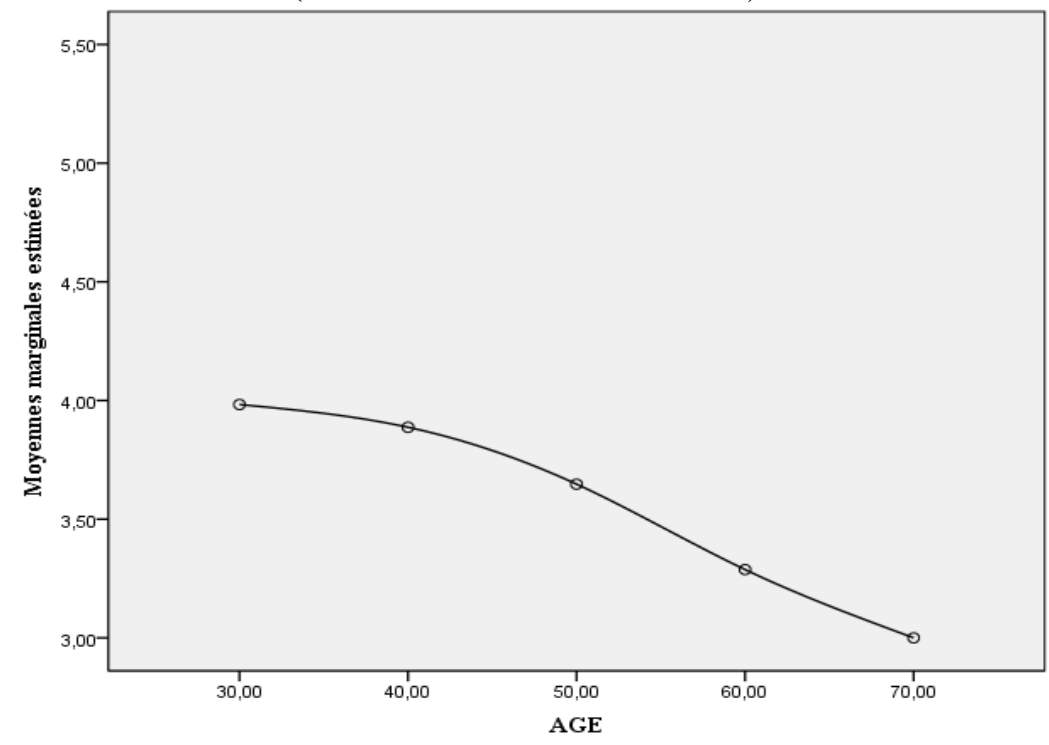


Figure 4 : Effet marginal de l'attitude sur l'intention entrepreneuriale à chaque âge (Intervalle de confiance : $95 \%$ )

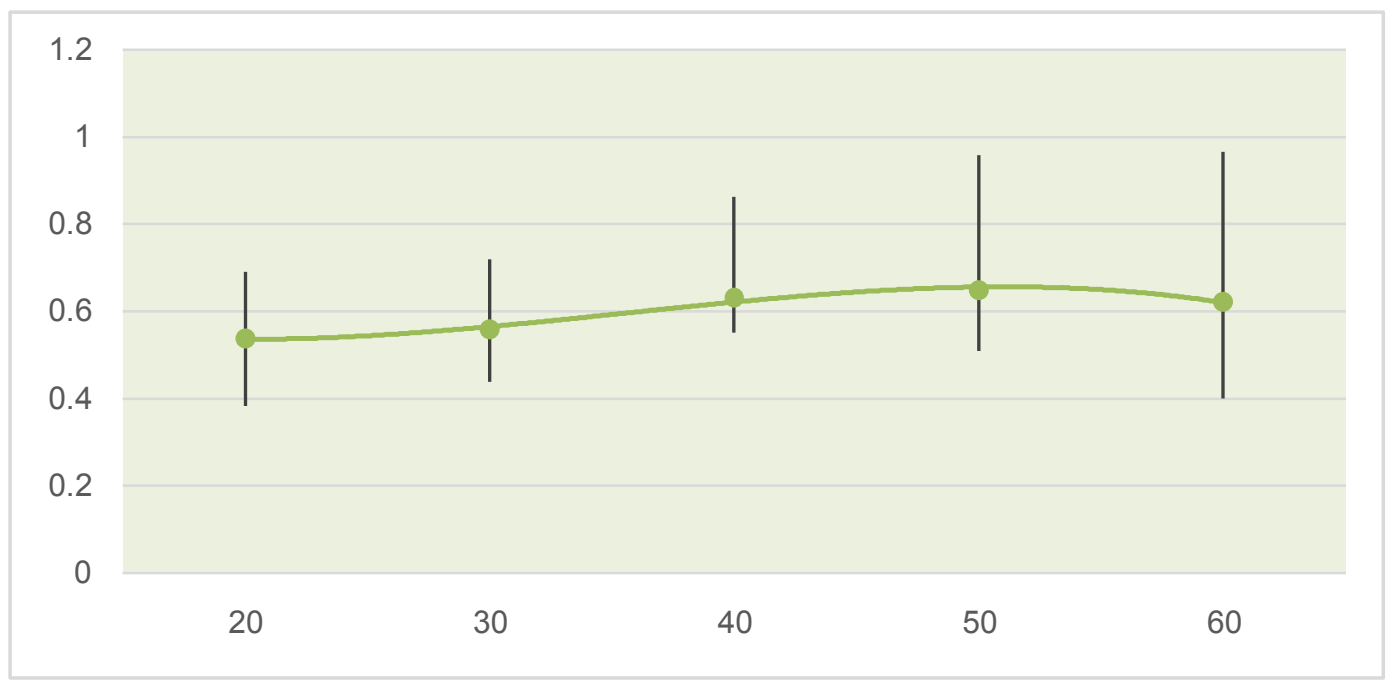

Figure 5 : Effet marginal de la faisabilité générale perçue sur l'intention entrepreneuriale à chaque âge (Intervalle de confiance : $95 \%$ )

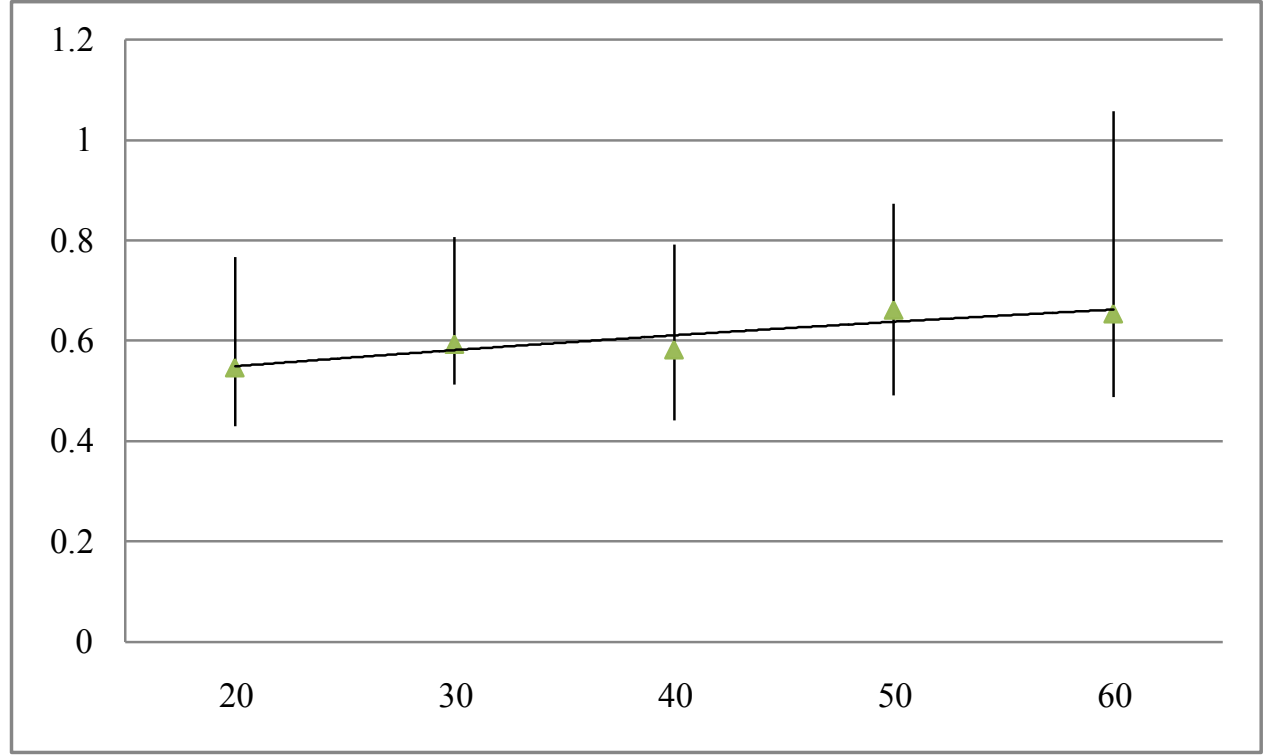

Les résultats de l'analyse statistique montrent qu'il y a des corrélations importantes autour de la variable modératrice l'âge. En effet, il y a une relation linéaire décroissante entre l'âge et l'intention entrepreneuriale, cela signifie que l'importance de l'intention entrepreneuriale diminue avec l'accroissement de l'âge. Cette confirmation est déduite du tableau 2 de la régression et du calcul de l'effet marginal de l'âge sur l'intention entrepreneuriale du figure 3.l'intention. Ce résultat obtenu rejoint parfaitement plusieurs études au préalablement effectuées (Rotefoss et Kolvereid, 2005) ${ }^{16}$.

Le tableau 2 nous montre que l'intention entrepreneuriale est influencée par l'attitude et le contrôle perçu.Par contre, la relation entre les normes subjectives et l'intention entrepreneuriale n'est pas significative. Cette

\footnotetext{
${ }^{16}$ Rotefoss, B., Kolvereid, L., 2005. Aspiring, nascent and fledgling entrepreneurs: an investigation of the business start-up process. Entrepreneurship \& Regional Development 17, 109
} 
constatation rejoint les travaux effectués par (Schlaegel et Koenig, 2014) ${ }^{17}$, qui considèrent que la désirabilité et le contrôle perçu sont les meilleurs prédicteurs de l'intention entrepreneuriale. L'impact de l'attitude sur l'intention diminue avec l'âge. En effet, l'intention entrepreneuriale est plus guidée par l'attitude chez les jeunes que chez les individus plus âgés. Ce résultat montre que le désir à entreprendre est relativement élevé chez les individus de moins de 45 ans (la génération Y), comparativement aux individus de plus de 45 ans (la génération Y).

Par contre, la relation entre le contrôle perçu et l'intention entrepreneuriale devient plus forte avec l'augmentation de l'âge (Figue 5). Ce qui montre que l'intention entrepreneuriale est moins guidée par le contrôle perçu chez les jeunes que chez les seniors. Ces résultats permettent de soutenir les hypothèses H1 et H2, autrement dit, les seniors sont plus guidés par le contrôle perçu que par l'attitude envers le comportement entrepreneurial.

\section{Conclusion:-}

Notre papier de recherche a pour objectif de mesurer l'impact de l'âge sur l'intention entrepreneuriale, en se basant sur la théorie du comportement planifié, sur les cadres salariés de la région Souss Massa.. L'étude empirique est effectuée sur un échantillon de 302 salariés exerçant dans des secteurs différents. Les résultats provenant de cette étude confirment que l'âge a un impact considérable sur l'intention entrepreneuriale. Ils montrent aussi que l'âge a un rôle modérateur entre l'attitude envers le comportement et le contrôle perçu qui sont deux composantes principales de la théorie du comportement planifié. En effet, l'intention entrepreneuriale est plus orientée par l'attitude envers le comportement entrepreneurial chez les jeunes (moins de 45 ans, génération $Y$ ) que chez les individus plus âgées (plus de 45 ans, génération X), par contre, l'intention entrepreneuriale des seniors est plus pilotée par le contrôle perçue. Les résultats montrent aussi que la relation entre les normes subjectives et l'intention entrepreneuriale n'est pas significative.

Notre apport théorique dans cette recherche est la prise en considération de la variable âge comme facteur important dans la formation de l'intention entrepreneuriale chez les salariés. Il existe de rares travaux au Maroc qui ont expliqués l'impact modérateur de 1 'âge entre la formation de l'intention entrepreneuriale et ses trois variables principales : attitude envers le comportement, le contrôle perçu et les normes subjectives.

Les jeunes salariés semblent plus motivés par leurs attitude envers le comportement pour la formation de l'intention entrepreneuriale. Il serait alors nécessaire de leur donner un contexte favorable à l'épanouissement de cette intention entrepreneuriale. Il faut penser alors aux outils pédagogiques managériales leur permettant de s'intégrer facilement dans le monde de création des entreprises.

Pour les futures recherches, Il serait très important de procéder à une analyse longitudinale permettant de faire un suivi des salariés ayant déclarés avoir l'intention entrepreneuriales pour savoir si ils sont passés réellement à l'acte entrepreneurial. Et finalement, on peut penser à introduire d'autres variables personnelles par exemple, la propension à la prise de risque et l'environnement économique entrepreneurial pour mieux comprendre les effets interactifs et élargir le champ de recherche.

\section{Bibliographie:-}

1. Ajzen, I., 1991. The theory of planned behavior. Organizational Behavior and Human Decision Processes 50, 179211. doi:10.1016/0749-5978(91)90020-T

2. Ajzen, I., Fishbein, M., 2004. Questions Raised by a Reasoned Action Approach: Comment on Ogden (2003). Health Psychology 23, 431-434.

3. Autio, E., Kenney, M., Mustar, P., Siegel, D., Wright, M., 2014. Entrepreneurial innovation: The importance of context. Research Policy 43, 1097-1108.

4. Bechard JP., comprendre le champ de l'entrepreneurship, cahier de recherches 96-01-01, HEC Montréal,1996

5. Ben Haddou, A., 1997. Maroc : les élites du Royaume, essai sur l'organisation du pouvoir au Maroc, L'Harmattan. ed.

6. Bourhis, A., Chenevert, D., 2010. Attraction et rétention des salariés du commerce de l'alimentation: Étude des salariés de la génération Y peu scolarisés. Presented at the XXIème Congrès de l'AGRH, Saint Malo.

7. Capiez A., les chances de succès des petites entreprises : vers un diagnostic d'émergence, revue internationale PME, 5 :2,1992,pp.103-132

\footnotetext{
${ }^{17}$ Schlaegel, C., Koenig, M., 2014. Determinants of Entrepreneurial Intent: A Meta-Analytic Test and Integration of Competing Models. Entrepreneurship: Theory \& Practice 38, 291.
} 
8. Cerdin, J.-L., Colle, R., Peretti, J.M., 2005. La fidélisation des salariés par l'entreprise à la carte. Revue de Gestion des Ressources Humaines 55, 2-21.

9. Degeorge, J.-M., Fayolle, A., 2011. Les étudiants français ont-ils la fibre entrepreneuriale? Entreprendre \& Innover 910, 21-28. doi:10.3917/entin.009.0021

10. Fairlie, R.W., 2004. Does Business Ownership Provide a Source of Upward Mobility for Blacks and Hispanics? Entrepreneurship and Public Policy, ed., Doug Holtz-Eakin, Cambridge: MIT Press.

11. Fayolle, A., Degeorge, J.M., Filion, L.J., 2012. Dynamique entrepreneuriale: Le comportement de l'entrepreneur, Petites Entreprises \& Entrepreneuriat. De Boeck Supérieur.

12. Fayolle, A., Gailly, B., 2015. The Impact of Entrepreneurship Education on Entrepreneurial Attitudes and Intention: Hysteresis and Persistence. Journal of Small Business Management 53, 75-93.

13. Fayolle, A., Liñán, F., 2014. The future of research on entrepreneurial intentions. Journal of Business Research 67 , 663-666. doi:10.1016/j.jbusres.2013.11.024

14. Fini, R., Grimaldi, R., Marzocchi, G.L., Sobrero, M., 2012. The Determinants of Corporate Entrepreneurial Intention Within Small and Newly Established Firms. Entrepreneurship Theory and Practice 36, 387-414.

15. Kautonen, T., Hatak, I., Kibler, E., Wainwright, T., 2015. Emergence of entrepreneurial behaviour: The role of agebased self-image. Journal of Economic Psychology 50, 41-51.

16. Kolvereid, L., 1996. Prediction of employment status choice intentions. Entrepreneurship: Theory \& Practice $21,47$.

17. Krueger, N.F., Reilly, M.D., Carsrud, A.L., 2000. Competing models of entrepreneurial intentions. Journal of Business Venturing 15, 411-432. doi:10.1016/S0883-9026(98)00033-0

18. Krueger, N.F., Carsrud, A.L., 1993. Entrepreneurial intentions: Applying the theory of planned behaviour. Entrepreneurship \& Regional Development 5, 315

19. Lee, L., Wong, P.K., Foo, M.D., Leung, A., 2011. Entrepreneurial intentions: The influence of organizational and individual factors. Journal of Business Venturing 26, 124-136. doi:10.1016/j.jbusvent.2009.04.003

20. Likert, R., 1932. A technique for the measurement of attitudes. Archives of Psychology 22, 1-55.

21. Maâlaoui, A., Fayolle, A., Castellano, S., Rossi, M., Safraou, I., 2012. L'entrepreneuriat des seniors. Revue française de gestion 227, 69-80. doi:10.3166/RFG.227.69-80

22. Maes, J., Leroy, H., Sels, L., 2014. Gender differences in entrepreneurial intentions: a TPB multi-group analysis at factor and indicator level. European Management Journal 32, 784-794.

23. Minniti, M., Lévesque, M., 2010. Entrepreneurial types and economic growth. Journal of Business Venturing 25, 305314.

24. Ogden, J. [b1] (analytic), 2003. Some problems with social cognition models: A pragmatic and conceptual analysis (English). Health psychology (Hillsdale, N.J.) 22, 424-428.

25. Pichault, F., Nizet, J., 2000. Les pratiques de gestion des ressources humaines. Approches contingente et politique,. Édition du Seuil, Paris.

26. Podsakoff, P.M., MacKenzie, S.B., Lee, J.Y., Podsakoff, N.P., 2003. Common method biases in behavioral research: a critical review of the literature and recommended remedies. Journal of Applied Psychology 88, 879-903.

27. Rotefoss, B., Kolvereid, L., 2005. Aspiring, nascent and fledgling entrepreneurs: an investigation of the business startup process. Entrepreneurship \& Regional Development 17, 109.

28. Safraou, I., Castellano, S., Maalaoui, A., Menvielle, L., 2012. Singularité du processus entrepreneurial chez les seniors. La Revue des Sciences de Gestion 255-256, 59-66. doi:10.3917/rsg.255.0059

29. Sammut S., Comment aider les petites entreprises jeunes ? Revue française de gestion, 121,1998b, pp.28-41.

30. Schlaegel, C., Koenig, M., 2014. Determinants of Entrepreneurial Intent: A Meta-Analytic Test and Integration of Competing Models. Entrepreneurship: Theory \& Practice 38, 291.

31. Scheiner, C., 2009. Fundamental Determinants of Entrepreneurial Behaviour, in: Fundamental Determinants of Entrepreneurial Behaviour. Gabler, pp. 5-44.

32. Thietart, R.-A., 2014. Méthodes de recherche en management, 4ème ed. Dunod.

33. Thompson, E.R., 2009. Individual entrepreneurial intent: Construct clarification and development of an internationally reliable metric. Entrepreneurship Theory and Practice 33, 669-694.

34. Tornikoski, E.T., Kautonen, T., Le Loarne, S., 2012. Le rôle de l'âge dans l'intention entrepreneuriale. Revue française de gestion 227, 95-109

35. van Gelderen, M., Brand, M., van Praag, M., Bodewes, W., Poutsma, E., van Gils, A., 2008. Explaining entrepreneurial intentions by means of the theory of planned behaviour. Career Development International 13, 538559

36. Van Stel, A., Carree, M., Thurik, R., 2005. The Effect of Entrepreneurial Activity on National Economic Growth. Small Business Economics 24, 311.

37. Wennekers, S., van Stel, A., Carree, M., Thurik, R., 2010. The Relationship between Entrepreneurship and Economic Development: Is It U-Shaped? Foundations \& Trends in Entrepreneurship 6, 167. 